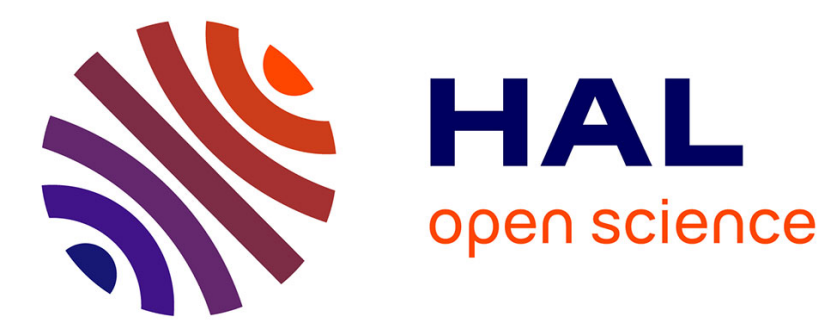

\title{
Interpretative transport modeling of the WEST boundary plasma: main plasma and light impurities
}

A. Gallo, A. Sepetys, Y. Marandet, H. Bufferand, G. Ciraolo, N. Fedorczak, S. Brezinsek, J. Bucalossi, J. Coenen, F. Clairet, et al.

\section{- To cite this version:}

A. Gallo, A. Sepetys, Y. Marandet, H. Bufferand, G. Ciraolo, et al.. Interpretative transport modeling of the WEST boundary plasma: main plasma and light impurities. Nuclear Fusion, 2020, 60 (12), pp.126048. 10.1088/1741-4326/abb95b . hal-03032714

\section{HAL Id: hal-03032714 https://hal.science/hal-03032714}

Submitted on 1 Dec 2020

HAL is a multi-disciplinary open access archive for the deposit and dissemination of scientific research documents, whether they are published or not. The documents may come from teaching and research institutions in France or abroad, or from public or private research centers.
L'archive ouverte pluridisciplinaire HAL, est destinée au dépôt et à la diffusion de documents scientifiques de niveau recherche, publiés ou non, émanant des établissements d'enseignement et de recherche français ou étrangers, des laboratoires publics ou privés. 


\title{
Interpretative transport modeling of the WEST boundary plasma. Part I: main plasma and light impurities.
}

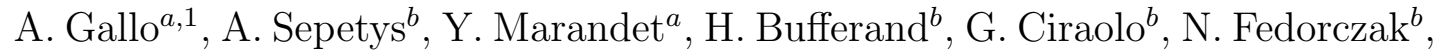 \\ S. Brezinsek ${ }^{c}$, J. Bucalossi ${ }^{b}$, J. Coenen $^{c}$, F. Clairet ${ }^{b}$, Y. Corre ${ }^{b}$, C. Desgranges ${ }^{b}$, \\ P. Devynck ${ }^{b}$, J. Gaspar ${ }^{d}$, R. Guirlet ${ }^{b}$, J. Gunn $^{b}$, C.C. Klepper ${ }^{e}$, J.-Y. Pascal ${ }^{b}$, \\ P. Tamain ${ }^{b}$, E. Tsitrone ${ }^{b}$, E.A. Unterberg ${ }^{e}$ and the WEST team ${ }^{2}$. \\ ${ }^{a}$ Aix-Marseille Univ, CNRS, PIIM, UMR 7345, Marseille, France \\ ${ }^{1}$ Current address: Oak Ridge Associated Universities, Oak Ridge, TN, USA \\ ${ }^{b}$ IRFM, CEA-Cadarache, Saint-Paul-lez-Durance, France \\ ${ }^{c}$ Forschungszentrum Jülich GmbH, Institut für Energie- und Klimaforschung- \\ Plasmaphysik, Jülich, Germany \\ ${ }^{d}$ Aix-Marseille Univ, CNRS, IUSTI, UMR 7343, Marseille, France \\ ${ }^{e}$ Oak Ridge National Laboratory, Oak Ridge, TN, USA \\ ${ }^{2}$ http://west.cea.fr/WESTteam
}

\section{Abstract}

Understanding impurity transport in tokamak plasmas is crucial to control radiative losses and material migration in future magnetic fusion reactors. In this work we deploy the SolEdge2D-EIRENE code to model the boundary plasma in a WEST discharge, satisfactorily reproducing measurements of both upstream and divertor plasma conditions. The spatial distribution of oxygen, studied here as a representative light impurity, is compared to vacuum ultraviolet spectroscopy measurements acquired with an oscillating line of sight. The simulation captures a key feature of the experiment, namely a factor of $\simeq 2$ higher oxygen brightness in the inner divertor region compared to the outer one. This spatial asymmetry in oxygen concentration is interpreted by analyzing the balance of friction forces and thermal gradient forces that the light impurity exchanges with the main plasma. 


\section{Introduction: studying the impact of light impurities in the WEST boundary plasma}

Future magnetic nuclear fusion reactors will have to rely on metallic plasmafacing components (PFCs) in order to sustainably handle long-term tritium retention and neutron-induced material damage [1]. The operation of these devices will be limited by plasma dilution and radiative losses due to both low and high $\mathrm{Z}$ impurities. In fact, impurities play a key role since they control the magnitude and the location of radiative losses. These losses can be beneficial in the boundary plasma as they help dissipating power loads onto the PFCs, but also deleterious in the core plasma as they reduce its temperature and the fusion reaction rate. The latter is of particular concern for fusion devices equipped with PFCs made of high $\mathrm{Z}$ materials, such as tungsten (W): such heavy impurities, eroded from the PFCs, can penetrate in the core plasma while being incompletely ionised, causing strong radiative losses. Therefore, a thorough understanding of the impurity transport in metallic tokamaks is needed to optimize and analyze the plasma scenarios of existing machines, as previously done in ASDEX Upgrade [2] and JET [3], as well as to prepare those of future ones.

The full-W tokamak WEST [4] is well fitted to study the impact of intrinsic light impurities, such as oxygen $(\mathrm{O})$, on the erosion and transport of $\mathrm{W}$. This can be done with the help of numerical models like the 2D multi-fluid plasma solver SolEdge2D $[5,6]$. In this contribution SolEdge2D, coupled with the Monte Carlo neutrals code EIRENE [10], is used to reproduce the deuterium (D) main plasma (section 3) and to study the spatial distribution of $\mathrm{O}$ as a representative light impurity (section 4) in a well characterized WEST plasma discharge (section 2). In particular, we compare our simulation with experimental data obtained via the WEST vacuum ultraviolet (VUV) spectroscopy system [11] with a moving line of sight in the poloidal plane, allowing one to retrieve the angular distribution of $\mathrm{O}$ light emission. These findings are analyzed and discussed in terms of thermal gradient and friction forces exerted by main ions and electrons on light impurity ions. The latter play a dominant role in setting the poloidal distribution of incident impurity fluxes onto the various PFCs. These fluxes rule the sputtering of heavy impurities which can then sustantially increase their content due to self-sputtering and migrate towards the confined plasma. A companion paper [12] studies the contamination pathways for heavy impurities for the very 
same WEST plasma discharge by comparing VUV measurements of molybdenum line emission with the results of numerical simulations performed with the ERO2.0 code [13]. These two papers should be seen as a unique effort showing that we can now provide valuable support to the interpretation of the role of impurities in present and future WEST operations. Our approach is enabled by the rapidly growing availability of WEST experimental data and by the synergistic deployment of state-of-the-art modeling tools, with the primary goal of understanding the core plasma contamination to improve the performance of the device.

\section{Experimental assessment of oxygen spatial distribution in the WEST divertor plasma}

To conduct this investigation we analyze a particular WEST plasma discharge (\#54067) performed during its third experimental campaign. This discharge is well diagnosed and offers the opportunity for a thorough comparison with our numerical modeling tools, as later presented in section 3. In general, WEST represents a good test bed for boundary plasma modeling in virtue of the simple open divertor geometry of its vacuum vessel and its set of dedicated edge plasma diagnostics [4]. In particular, the considered plasma discharge is characterized by stable L-mode conditions and, during a 4 s flattop phase, benefits from a unique feature of its VUV spectroscopy system [11], which can scan the lower half of the plasma thanks to a sweeping line of sight. This is done by moving the spectrometer mechanically up and down with a period of a few seconds. When used in this scanning mode, the system provides spatial profiles of spectral line emission from which information can be deduced about the emitting ion spatial distribution. In the VUV spectra of discharge \#54067, several OIII and OIV lines are identified, thus allowing the comparison of the poloidal distribution of $\mathrm{O}^{2+}$ and $\mathrm{O}^{3+}$ lines found experimentally with the one simulated by SolEdge2D-EIRENE, as described in section 4 . The main machine parameters during this discharge are: deuterium (D) as fuelling gas, lower single null (LSN) magnetic configuration (shown in figure 2.b), X-point to lower divertor distance $d X=115 \mathrm{~mm}$, toroidal magnetic field $B_{\mathrm{T}}=3.8 \mathrm{~T}$, plasma current $I_{\mathrm{P}}=500 \mathrm{kA}$ throughout the entire $10 \mathrm{~s}$ duration of the discharge. In terms of power balance, during the steady state L-mode phase between $6 \mathrm{~s}$ and $9 \mathrm{~s}$, the most impor- 
tant parameters, shown in figure 1.a, are: Ohmic power $P_{\mathrm{ohm}} \simeq 0.1 \mathrm{MW}$, power from lower hybrid current drive (LHCD) $P_{\mathrm{LHCD}} \simeq 4.2 \mathrm{MW}$, total heating power $P_{\text {heat }} \simeq 4.3 \mathrm{MW}$, power radiated in the bulk plasma (defined in section 3.3) measured by bolometers $P_{\text {rad }}^{\text {bulk }} \simeq 1.6 \mathrm{MW}$, power losses due to magnetic ripple $P_{\text {ripple }}=0.1 P_{\mathrm{LHCD}} \simeq 0.4 \mathrm{MW}$. The power entering the scrape-off layer (SOL) can, therefore, be estimated from a power balance as $P_{\mathrm{SOL}}=P_{\text {heat }}-P_{\mathrm{rad}}^{\text {bulk }}-P_{\text {ripple }} \simeq 2.3 \mathrm{MW}$. The line integrated density measured by the interfero-polarimeter system [14], shown in figure 1.b, is $n_{\text {lid }}^{\text {main }}=4 \times 10^{19} \mathrm{~m}^{-2}$ in the main plasma, whereas in the lower divertor it is $n_{\text {lid }}^{\text {div }}=3-4 \times 10^{18} \mathrm{~m}^{-2}$, with a $\simeq 30 \%$ increase during this phase. This is a commonly observed behaviour in WEST discharges performed in LSN configuration, possibly due to the outgassing of D atoms from the PFCs as their temperature rises.
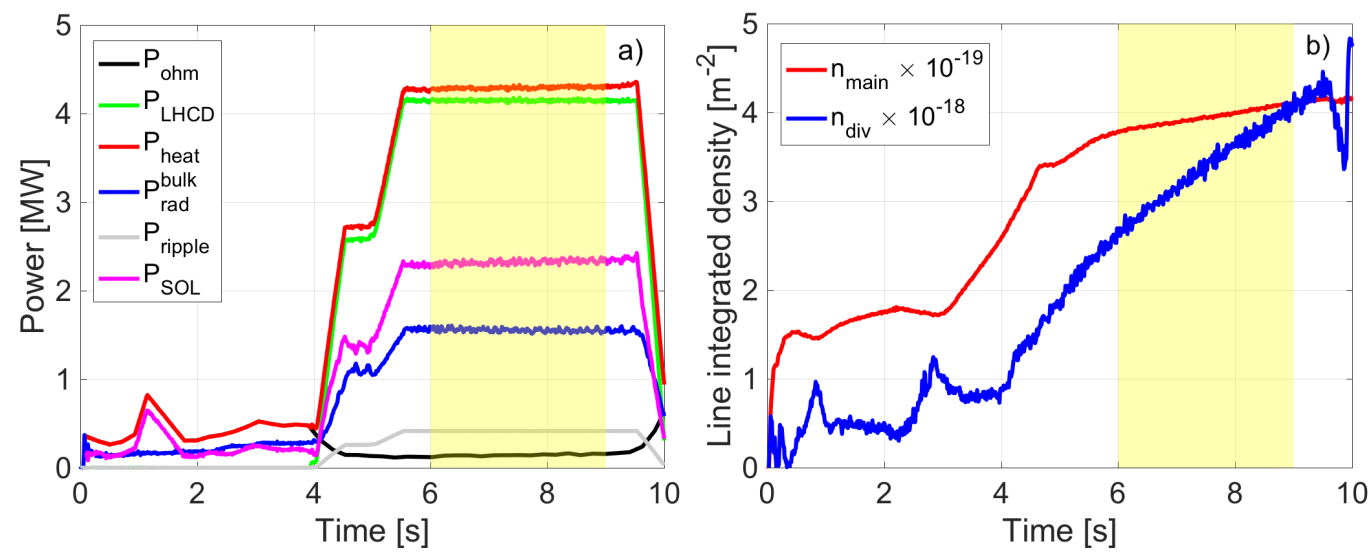

Figure 1: a) time traces for the WEST plasma discharge \#54067: Ohmic power (black), lower hybrid current drive power (green), total heating power (red), power radiate in bulk plasma (blue), power losses due to magnetic ripple (grey), power entering the scrape-off layer (magenta); b) time traces of the main plasma (red) and divertor (blue) line integrated density; the yellow shaded area corresponds to the VUV spectroscopy scan time interval. 


\section{Modeling of the main plasma}

\subsection{Model, setup and assumptions}

The WEST plasma discharge \#54067 described in section 2 is numerically modeled with our edge and scrape-off layer code suite: SolEdge2D-EIRENE [6]. The model, in the following referred to as S2DE, consists in the coupling of the 2D multi-fluid plasma solver SolEdge2D [5] and the kinetic Monte Carlo neutral code EIRENE [10]. Thanks to its immersed boundary condition, the so-called penalization technique, S2DE can be used to calculate density, temperature and velocity of electrons and multiple ion species in realistic tokamak geometry. As sketched in figure 2.a, the simulation domain (green area) extends from an inner boundary located at a normalized minor radius $\rho=0.823$ in the edge plasma region (blue solid line) down to the wall contour (black solid line) accounting for in-vessel plasma-facing components (PFCs) as, for instance, the baffle and the LHCD antenna. The latter, however, has a finite toroidal extent $\left(\simeq 30^{\circ}\right)$ and a complex geometrical structure which cannot be accurately described by a 2D model. This antenna, which can be moved radially in experiments, is positioned in the simulation at a location where the level of recycling on its surface is low enough to be consistent with visible camera observations (i.e., no recycling detected there). The ability to calculate plasma fluxes on main chamber structures, such as the baffle, is a strong asset for S2DE in the panorama of interpretative 2D transport models, especially if the focus is on plasma-wall interactions and core contamination studies.

The S2DE mesh is based on the magnetic equilibrium for discharge \#54067 for $t=8 \mathrm{~s}$ obtained with the real time plasma shape reconstruction tool VacTH $[15,16]$. For the simulation presented in this paper, the output of the VacTH calculation is modified to account for two experimental evidences. i) The top part of the magnetic equilibrium given by VacTH (figure 2.b, red contours) has a high upper triangularity. This feature leads to strong particle and heat fluxes at the upper divertor targets in S2DE simulations. Such fluxes are not backed by Langmuir probe measurements of ion saturation current $j_{\text {sat }}$. Moreover, the high recycling at the upper divertor observed in simulations, stronger than at the lower divertor, is not supported by visible light imaging data. ii) The location of the strike points at the lower divertor targets given by VacTH shows a disagreement with respect to the one inferred from embedded diagnostics like Langmuir probes, thermocouples, 
fiber Bragg gratings and from infrared thermography. The modified magnetic equilibrium adopted for this study (figure 2.b, blue contours), therefore, has an X-point vertical position lowered by $\simeq 15 \mathrm{~mm}$ and a distance between the strike points along the divertor targets reduced by $\simeq 25 \mathrm{~mm}$.
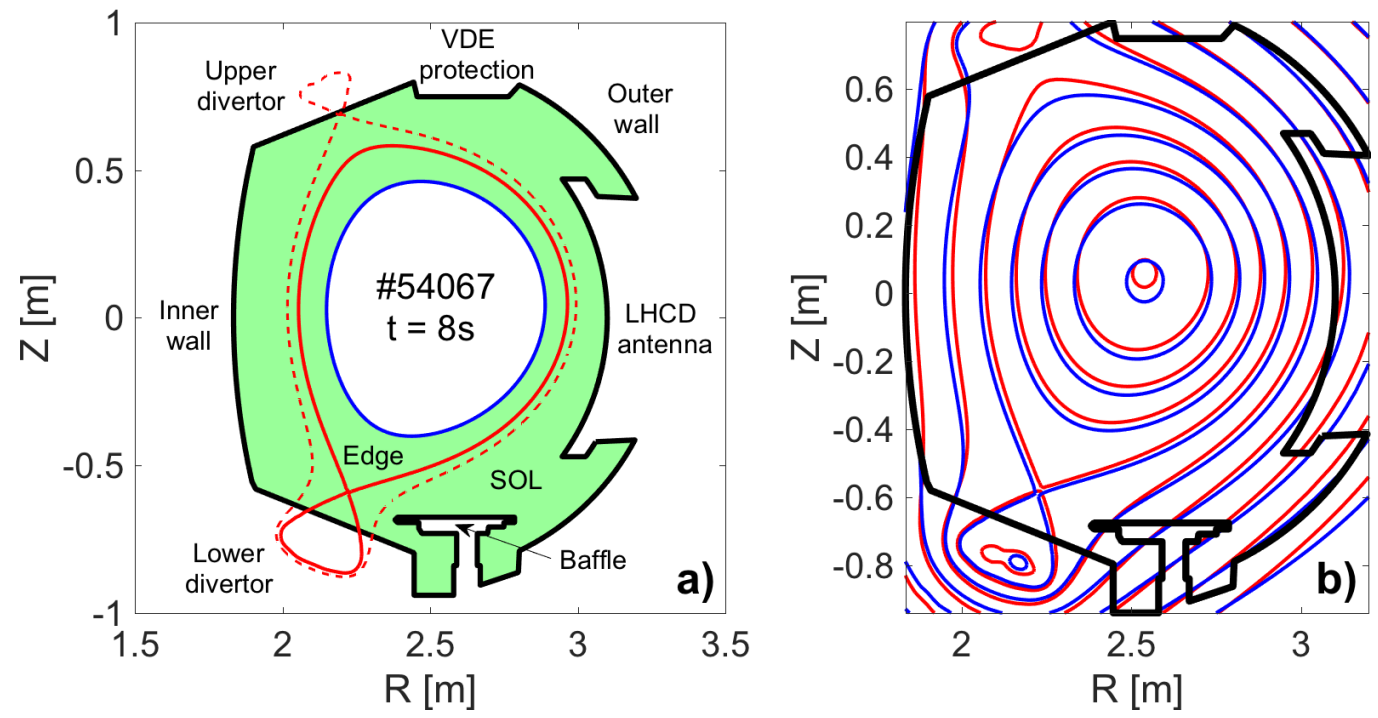

Figure 2: a) domain of the SolEdge2D-EIRENE simulation (green area), WEST wall contour with relevant plasma-facing components (black solid line), inner simulation boundary (blue solid line), main and secondary magnetic separatrix (solid and dashed red line); b) Magnetic flux contours for WEST discharge \#54067 at $t=8 \mathrm{~s}$ as calculated by VacTH (red lines) and modified with input from plasma diagnostics and simulations (blue lines).

We now discuss the main input parameters for the S2DE run, also summarized below in table 1 . The $\mathrm{D}$ ion density at the inner simulation boundary $n_{\mathrm{D}^{+}}^{\mathrm{IB}}=2.5 \times 10^{19} \mathrm{~m}^{-3}$ is chosen in order for the electron density at the same location to match the fast sweep reflectometer experimental data within the error bars $\left(n_{\mathrm{e}}^{\mathrm{IB}} \simeq 3 \times 10^{19} \mathrm{~m}^{-3}\right)$. We assume a total power entering the simulation domain at the inner boundary of $P_{\text {tot }}^{\mathrm{IB}}=2.3 \mathrm{MW}$, using $P_{\mathrm{SOL}}$ obtained from the power balance previously discussed in section 2; this total power is assumed to be equally shared between electrons and D ions, $P_{\mathrm{D}^{+}}^{\mathrm{IB}}=P_{\mathrm{e}}^{\mathrm{IB}}=1.15 \mathrm{MW}$. The total $\mathrm{O}$ density at the inner simulation boundary, which represents the initial impurity source in this simulation, is $n_{\mathrm{O}^{\mathrm{n}+}}^{\mathrm{IB}}=0.02 n_{\mathrm{D}^{+}}^{\mathrm{IB}}=5 \times 10^{17} \mathrm{~m}^{-3}$, where the superscript $\mathrm{n}+$ signifies sum over 
all $\mathrm{O}$ ionisation states from now on (excluding neutrals); this assumption is motivated by recent experimental studies of the visible spectroscopy data in the WEST lower divertor showing that an $\mathrm{O}$ content of $1-2 \%$ is consistent with both effective $\mathrm{W}$ sputtering yields and residual gas analysis [17]. Typical L-mode transport coefficients $[6,18]$ are used for particles and momentum $D=\nu=1 \mathrm{~m}^{2} \mathrm{~s}^{-1}$ as well as for energy $\chi=2 \mathrm{~m}^{2} \mathrm{~s}^{-1}$; these values, uniform over the whole domain and equal for all species, ensure a very good match of the main plasma profiles in the lower divertor with embedded diagnostics, as shown below. We adopt a recycling coefficient uniform along the full-W wall contour of $R=0.99$ and an albedo coefficient for the pump $A=0.9$, both coefficients equal for $\mathrm{D}$ and $\mathrm{O}$; these numbers represent the standard S2DE assumption for W PFCs and for the WEST pumping system, respectively. In steady state, recycled fluxes at the wall are the main sources of $\mathrm{D}$ and $\mathrm{O}$. It should be noted that magnetic drifts are turned off in this simulation: the potential consequences of this working hypothesis on the transport of light impurities in the divertor region are discussed at the end of the paper.

\begin{tabular}{|c|c|c|c|}
\hline Quantity & Value & Unit & Motivation \\
\hline \hline$n_{\mathrm{D}^{+}}^{\mathrm{IB}}$ & $2.5 \times 10^{19}$ & $\mathrm{~m}^{-3}$ & $n_{\mathrm{e}}^{\mathrm{IB}}$ to match fast sweeping reflectometer \\
\hline$P_{\text {tot }}^{\mathrm{IB}}$ & 2.3 & $\mathrm{MW}$ & Power balance (heating and bolometry) \\
\hline$n_{\mathrm{O}^{\mathrm{n}+}}^{\mathrm{IB}}$ & $5 \times 10^{17}$ & $\mathrm{~m}^{-3}$ & Visible spectroscopy (previous studies) \\
\hline$D, \nu$ & 1 & $\mathrm{~m}^{2} \mathrm{~s}^{-1}$ & Best match to divertor Langmuir probes \\
\hline$\chi$ & 2 & $\mathrm{~m}^{2} \mathrm{~s}^{-1}$ & Best match to probes and thermocouples \\
\hline$R$ & 0.99 & - & Standard assumption for W PFCs \\
\hline$A$ & 0.9 & - & Standard assumption for WEST pumps \\
\hline
\end{tabular}

Table 1: Main input parameters for the SolEdge2D-EIRENE modeling.

\subsection{Main plasma profiles along the outer midplane and poloidal distribution}

In this section we discuss the results concerning the main plasma, whereas the light impurity modeling results are presented in section 4 . Let us start with the outer midplane radial profiles for the main plasma density and temperature, shown in figure 3. In figure 3.a, the density profiles of electrons (blue line) and D ions (red line) simulated with S2DE are superimposed to experimental $n_{\mathrm{e}}$ data from the midplane fast sweeping reflectometer [19] 
(green dots with horizontal error bars) and the reciprocating Langmuir probe (RLP) [20] (cyan and magenta circles). In the edge plasma the profile of $n_{\mathrm{e}}$ calculated by S2DE matches reflectometry data within the $1.2 \mathrm{~cm}$ radial uncertainty, whereas it is higher by a factor of 1.8 at the main separatrix, and by a factor of 2.1 at the secondary separatrix. A quantitative agreement with reflectometry data throughout the entire $n_{\mathrm{e}}$ profile can be achieved by dividing the transport coefficient values by 2 (not shown). However, the simulation discussed here represents our best effort in simultaneously matching outer midplane and lower divertor target measurements, shown below, after scanning $n_{\mathrm{D}^{+}}^{\mathrm{IB}}, D, \nu, \chi$. On the other hand, the agreement on $n_{\mathrm{e}}$ from S2DE and RLP data (cyan circles) is poor. It is important to mention that this probe is located at the top of the machine, where the uncertainties affecting the magnetic equilibrium reconstruction are bigger: RLP data acquired in the vicinity of the secondary X-point are remapped at the outer midplane along magnetic flux surfaces, which can result in uncertainties in the radial direction. It is likely that the very low $n_{\mathrm{e}} \leq 2 \times 10^{17} \mathrm{~m}^{-3}$ measured by the RLP corresponds to a radial location further out in the SOL.
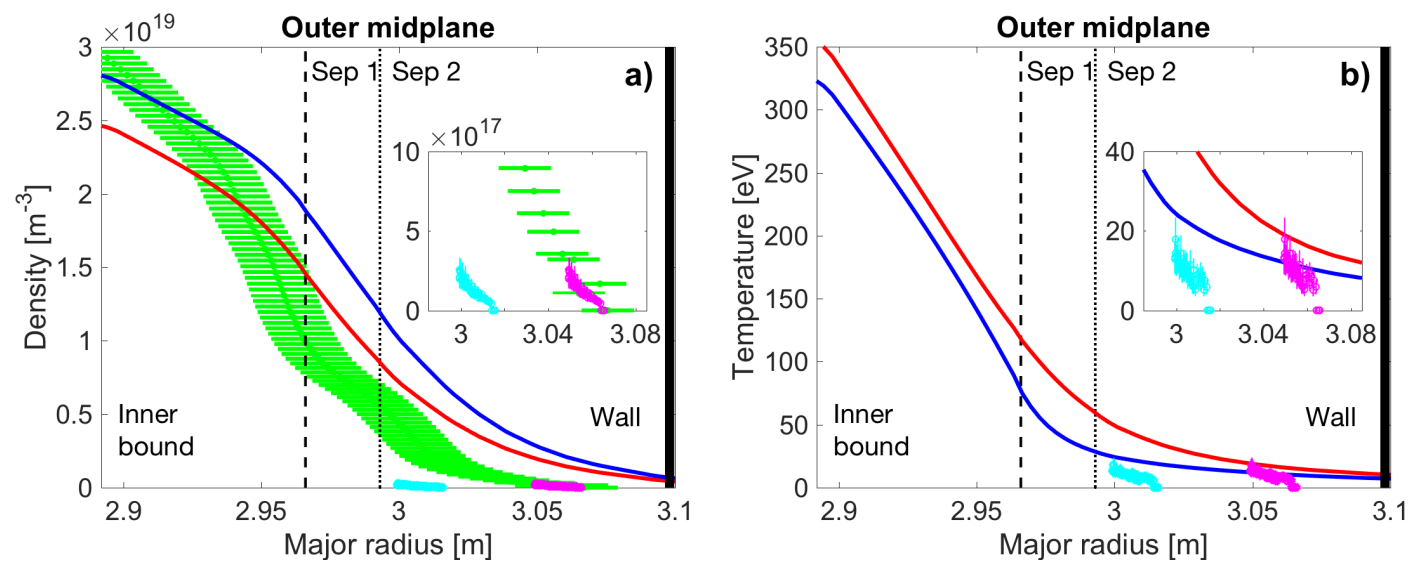

Figure 3: outer midplane radial profiles of a) density and b) temperature with main (dashed line) and secondary (dotted line) separatrix, wall contour (thick solid line). Solid curves for SolEdge2D-EIRENE simulations (blue for electrons, red for ions), green dots with horizontal error bars for fast sweeping reflectometer data, cyan (uncorrected) and pink (corrected) dots for reciprocating Langmuir probe data (remapped at the outer midplane).

By displacing the remapped RLP data by $5 \mathrm{~cm}$ outwards (magenta circles), 
the agreement with reflectometry data becomes quantitative, as shown in the inset. The same argument applies to the temperature profiles depicted in figure 3.b: although here only a factor of 2 to 4 difference is found between $T_{\mathrm{e}}$ from S2DE (blue line) and $T_{\mathrm{e}}$ from the RLP data (cyan circles), the agreement is greatly improved if the above mentioned $5 \mathrm{~cm}$ radial shift is applied (magenta circles), as can be seen in the inset. No other measurements are available at time of writing for further comparison with the S2DE profiles of $T_{\mathrm{e}}$ or $T_{\mathrm{i}}$ (red line). Nevertheless, the values of $T_{\mathrm{e}}^{\mathrm{sep}} \simeq 80 \mathrm{eV}$ and $T_{\mathrm{i}}^{\mathrm{sep}} \simeq 120 \mathrm{eV}$ found in the simulation, as well as temperature decay lengths of the order of few centimeters, are compatible with typical experimental findings in L-mode plasma conditions as well as with estimations from a simple two-point model [9].

The corresponding poloidal maps for $\mathrm{D}$ ion density $n_{\mathrm{D}^{+}}$and temperature $T_{\mathrm{D}^{+}}$are shown in figure 4.a and 4.b, respectively. Despite the considered plasma discharge being in LSN configuration, it is clear that the secondary separatrix (black dashed lines), the X-point of which lies close to the upper divertor surface, has a strong impact on SOL profiles with the assumed transport coefficients: the $n_{\mathrm{D}^{+}}$map shows values that are poloidally rather uniform in the edge and near SOL regions, with density in the lower and upper divertor regions of the same order of magnitude $\left(10^{19} \mathrm{~m}^{-3}\right)$; the $T_{\mathrm{D}^{+}}$ map, instead, shows a D plasma which is hotter in the low field side (LFS) SOL when compared to the high field side (HFS) SOL, where the D neutral pressure is higher (not shown).

\subsection{Radiation pattern and synthetic diagnosis}

The total radiated power in the $\mathrm{S} 2 \mathrm{DE}$ simulation $(\mathrm{D}+\mathrm{O})$ is $P_{\mathrm{rad}}^{\mathrm{tot}}=818 \mathrm{~kW}$, of which the fraction radiated in the $\mathrm{SOL}\left(P_{\mathrm{rad}}^{\mathrm{SOL}}=664 \mathrm{~kW}\right)$ is $f_{\mathrm{rad}}^{\mathrm{SOL}} \simeq 81 \%$. The power radiated by $\mathrm{D}$ accounts for $137 \mathrm{~kW}(\simeq 17 \%$ of the total) and the $\mathrm{D}$ radiation pattern is more strongly localised in the SOL $\left(f_{\mathrm{rad}}^{\mathrm{SOL}} \simeq 92 \%\right)$. As can be seen in the emissivity map found in figure 5.a, D radiates mainly in the immediate vicinity of the lower divertor targets (recycling) but important contributors are also the entire lower and upper divertor regions as well as the HFS near SOL. The power radiated by $\mathrm{O}$ is discussed in section 4 . The total radiated power measured by the WEST bolometry system for discharge \#54067 and averaged over the $t=6-9$ s plateau is $P_{\text {rad }}^{\text {tot }} \simeq 2.3 \mathrm{MW}$. All bolometry measurements are characterized by an experimental uncertainty of $10 \%$. Here the S2DE simulation cannot be directly compared to 

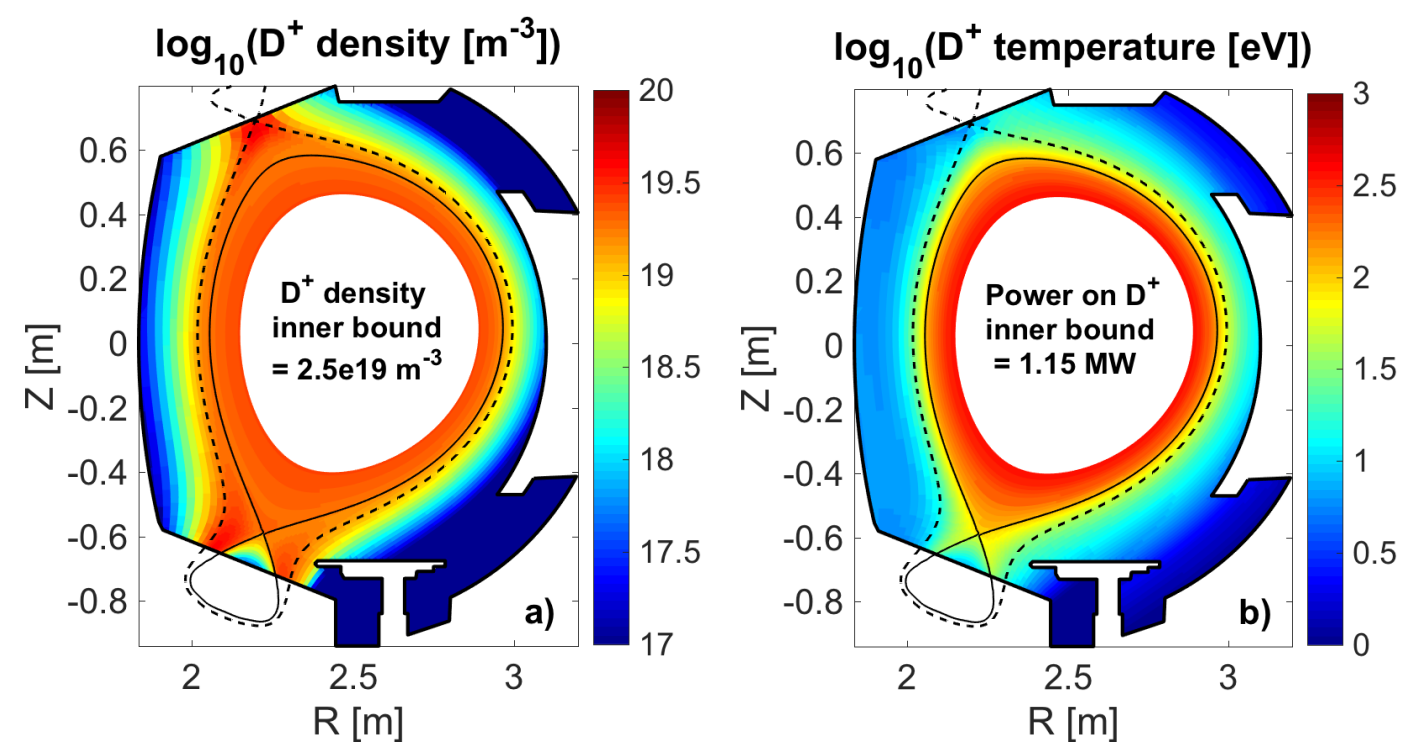

Figure 4: poloidal maps of deuterium ion a) density and b) temperature calculated by SolEdge2D-EIRENE with main (solid line) and secondary (dashed line) magnetic separatrix. The assumptions on deuterium ion density and power at the inner simulation boundary are recalled in the cutouts.

the bolometry measurement as the former does not include the contribution from the core plasma, which is the dominant one. Due to the geometry of the channels of the bolometry system, traced in figure 6.a, we can only separate the contributions of lower divertor, bulk plasma and upper divertor, which correspond to $P_{\text {rad }}^{\text {updiv }} \simeq 0.2 \mathrm{MW}, P_{\text {rad }}^{\text {bulk }} \simeq 1.6 \mathrm{MW}$ and $P_{\text {rad }}^{\text {lodiv }} \simeq 0.5 \mathrm{MW}$, respectively. It is found that the measured $P_{\text {rad }}^{\text {lodiv }}+P_{\text {rad }}^{\text {updiv }} \simeq 0.7 \mathrm{MW}$ is bigger than $P_{\text {rad }}^{\mathrm{SOL}}$ in S2DE, which accounts also for the contribution of the HFS and LFS edge and SOL. In this experimental setup, the power radiated in these regions cannot be disentangled from $P_{\text {rad }}^{\text {bulk }}$. Nevertheless, a quantitative comparison between the value measured along the channels comprised within the S2DE simulation domain is possible thanks to a newly developed SYNthetic DIagnostic: SYNDI is a Monte Carlo beam tracer for computing the power received by a synthetic detector due to the radiation emitted by the simulated plasma. The plasma radiation is prescribed as a 2D map of volume emissivity, assuming toroidal symmetry. The detector is defined as a section of plan (rectangle or circular), with arbitrary orientation and collects the plasma radiation through a diaphragm of arbitrary orientation. A 

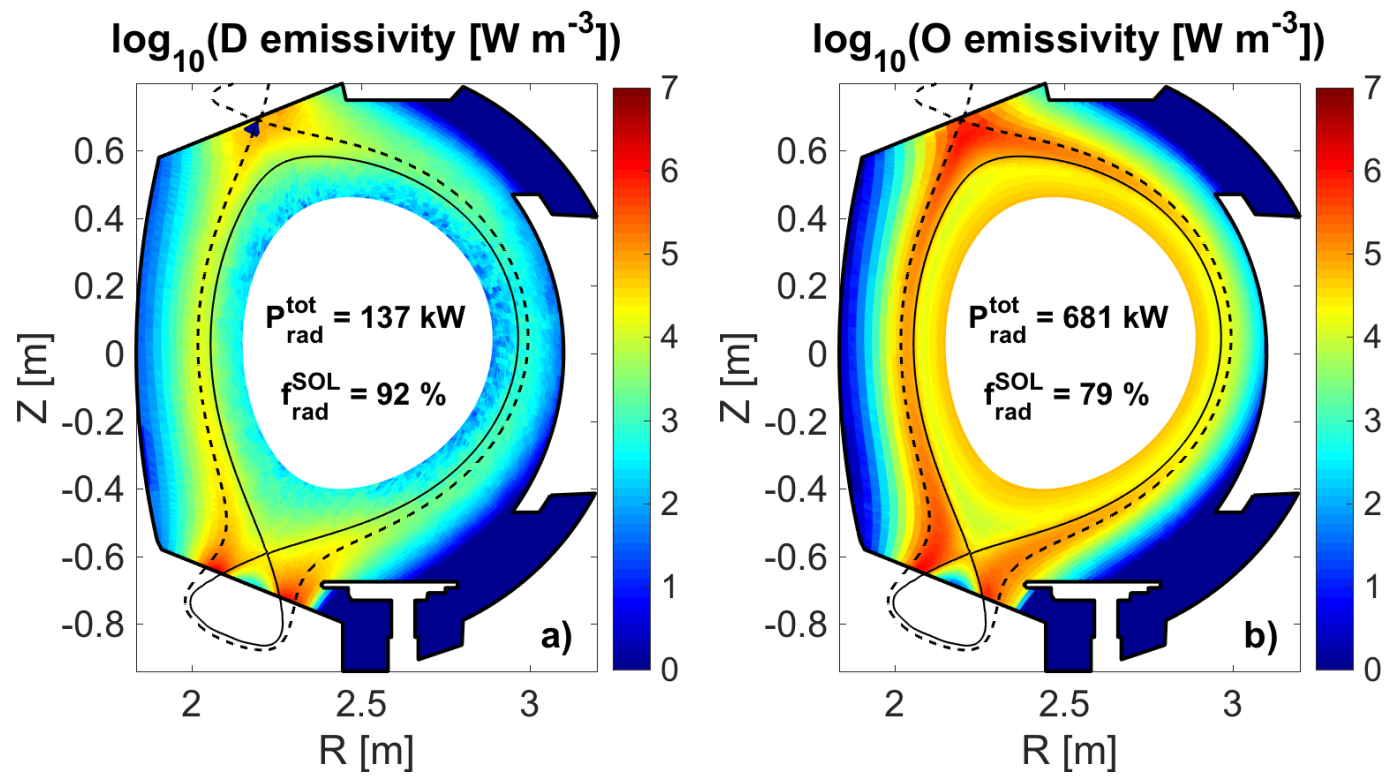

Figure 5: poloidal maps of emissivity for a) deuterium and b) oxygen calculated by SolEdge2D-EIRENE. The total radiated power $P_{\text {rad }}^{\text {tot }}$ and the fraction radiated in the scrape-off layer $f_{\text {rad }}^{\mathrm{SOL}}$ are reported in the cutouts.

light beam is defined from a random starting position on the detector, and a random passing position on the diaphragm. The beam path is then followed toward the plasma as a straight line, along which the volume emissivity is simply integrated. When the beam path reaches the first wall contour, it is either stopped or allowed to reflect with prescribed properties and probabilities. A prescribed number of beams are processed, line integrals of emissivity are averaged and finally multiplied by the geometrical extension of the detector/diaphragm system. The radiated power measured by the lower divertor bolometry channels 1, 2 and 3 (figure 6.c, black dots) is respectively 13.5, 3.5 and 2 times higher than the one found by SYNDI for the S2DE run (figure 6.c, red squares). This result suggests that the working assumptions behind our simulation lead to a strong underestimation of the baffle top and/or the far LFS SOL contributions (channel 1) but allow to reproduce reasonably well the power radiated in the lower outer divertor (channel 2) as well as the $\mathrm{X}$-point and lower inner divertor region (channel 3). A better agreement is found for the upper divertor channels 14, 15 and 16 (figure 6.b) where S2DE matches the measurements within a factor of 2.8, 0.64 and 1.7, respectively. 
Overall, the simulation seems to underestimate the radiated power suggesting that the assumption on the light impurity concentration might be too low with respect to the actual experimental conditions.
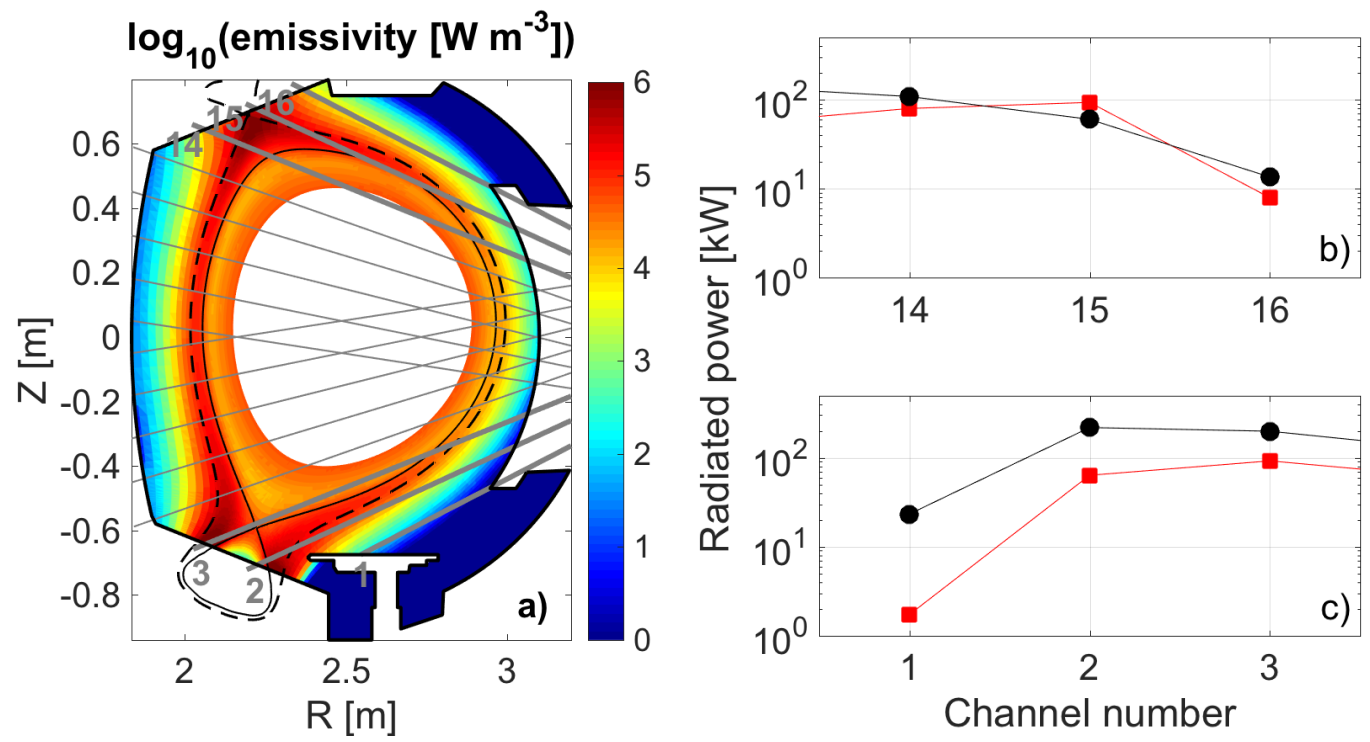

Figure 6: a) poloidal map of emissivity calculated by SolEdge2D-EIRENE with bolometry channels (grey lines); b) power radiated along channels 14 to 16 and c) along channels 1 to 3 from bolometers (black dots) and calculated by SolEdge2D-EIRENE (red squares).

\subsection{Main plasma profiles along the lower divertor}

Finally, in figure 7, the background plasma quantities at the lower divertor targets calculated by S2DE are compared with experimental data measured at $t=8 \mathrm{~s}$ by two embedded diagnostic systems of WEST: divertor Langmuir probes (DLP) [21] and thermocouples (TC) [22, 23]. Within the experimental uncertainties of $\simeq 30 \%$, a nearly quantitative agreement of $n_{\mathrm{e}}, T_{\mathrm{e}}$ and the parallel heat flux $q_{\|}$is found between DLP measurements (black circles) and the S2DE simulation (blue line), as shown in figures 7.a, 7.b and 7.d, respectively. In particular, concerning $n_{\mathrm{e}}$, DLP data suggest that the lower divertor plasma is in the conduction limited regime as the peak at both targets $\left(n_{\mathrm{e}}^{\text {div }}=3\right.$ to $\left.4 \times 10^{19} \mathrm{~m}^{-3}\right)$ is found to be higher than the average value measured at the outer midplane separatrix via reflectometry $\left(n_{\mathrm{e}}^{\text {omp }} \simeq\right.$ 
$1.5 \times 10^{19} \mathrm{~m}^{-3}$ ), shown above in figure 3.a. This observation is backed by the significant $T_{\mathrm{e}}$ gradient found by S2DE between the outer midplane $\left(T_{\mathrm{e}}^{\mathrm{omp}} \simeq\right.$ $80 \mathrm{eV})$ and the peak at both targets $\left(T_{\mathrm{e}}^{\text {div }} \simeq 40 \mathrm{eV}\right)$. The ion saturation current density $j_{\text {sat }}$ (figure 7.c) measured by DLP is reproduced by S2DE within the error bars at the inner divertor target. On the other hand, while the outer target profile is satisfactorily matched in the far SOL, the $j_{\text {sat }}$ values in the near SOL sit below the DLP error bars. This discrepancy could be due to the absence of mean drift flows, which would recirculate ions from the inner to the outer target, leading to an in/out asymmetry of the $j_{\text {sat }}$ profile closer to the experimental one. The agreement in $q_{\|}$between S2DE and DLP data is good, in terms of both absolute values and decay lengths. For the estimation of $q_{\|}$from DLP data, a sheath heat transmission coefficient $\gamma=8$ is adopted, therefore assuming hot ions $\left(T_{\mathrm{i}} \simeq 2 T_{\mathrm{e}}\right)$ and a pure $\mathrm{D}$ plasma [9]. Given the unavailability of $T_{\mathrm{i}}$ measurements at the time of writing, such an assumption can be refined by tuning $\gamma$ for each individual probe using the local $T_{\mathrm{i}} / T_{\mathrm{e}}$ ratio calculated from S2DE. In fact, the simulated $T_{\mathrm{i}} / T_{\mathrm{e}}$ at the lower divertor targets (not shown) is as low as $\simeq 0.7$ in the vicinity of the strike points but rises up to $\simeq 1.2$ in the far $\mathrm{SOL}$, leading to $\gamma_{\mathrm{S} 2 \mathrm{DE}}$ values in the range 6.3 to 7.2. The $q_{\|}$data from DLP are therefore recalculated for each probe (green circles in figure 7) using $\gamma=\gamma_{\mathrm{S} 2 \mathrm{DE}} / 8$, resulting in an even better agreement with S2DE, which is quantitative within error bars. Estimates of the same quantity coming from the TC system (red diamonds) further corroborate our observations at the outer divertor target, where TC, DLP and S2DE agree very nicely. On the other hand, at the inner divertor target, TC data present a peak $q_{\|}$smaller by factor of $\simeq 2$ than both uncorrected DLP data and S2DE calculation. This discrepancy between the two diagnostics, which is anyway reduced when considering the S2DE-corrected data, is not yet understood at the time of writing. Overall, a good agreement is obtained for both outer midplane and lower divertor main plasma parameters between the WEST boundary plasma diagnostics and the S2DE simulation under the above mentioned assumptions. 

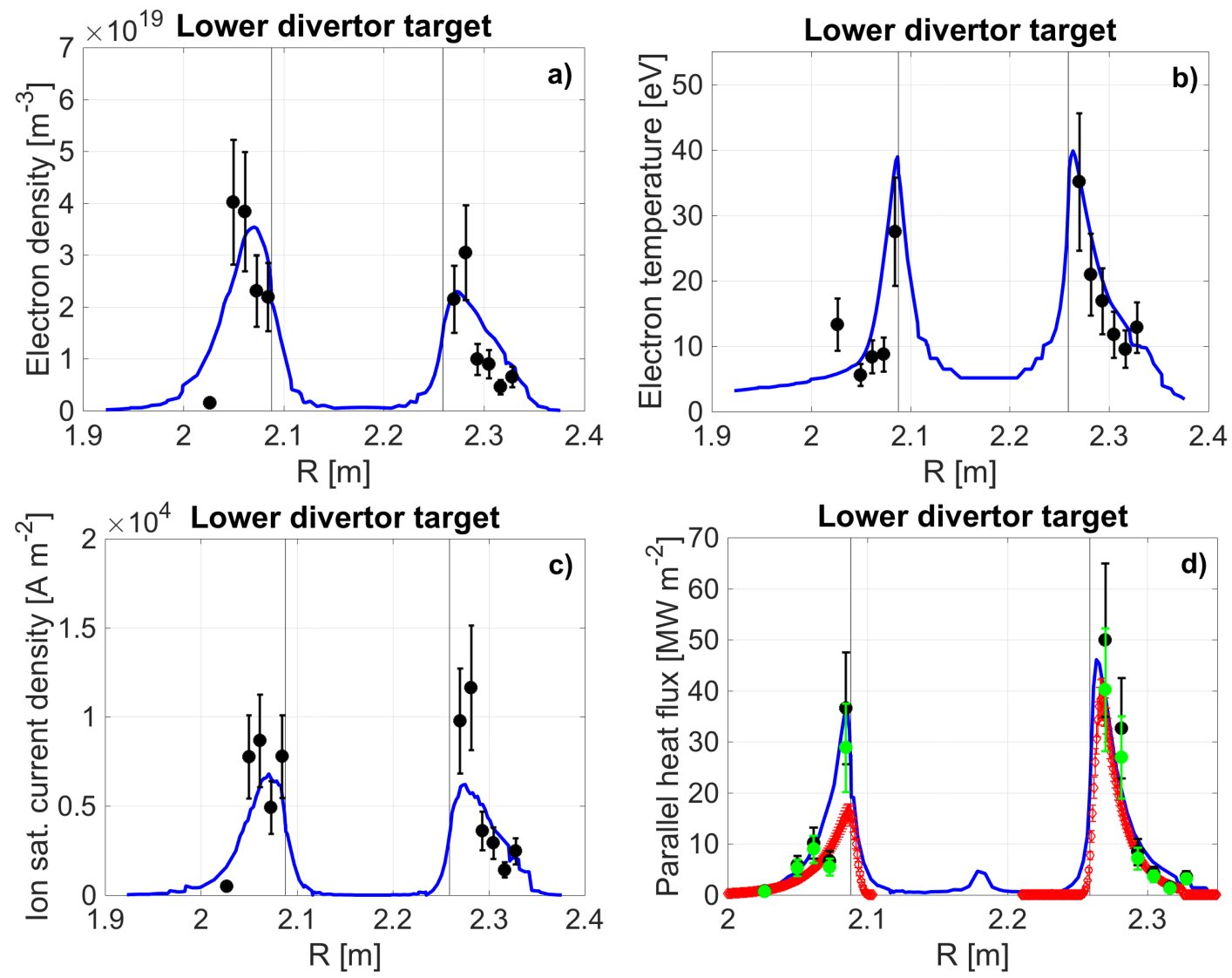

Figure 7: Radial profiles of a) electron density, b) electron temperature, c) ion saturation current density and d) parallel heat flux along the lower divertor. Blue lines for SolEdge2D-EIRENE simulation, black circles for divertor Langmuir probes data and red diamonds for thermocouples data. Green circles for divertor Langmuir probes data corrected with ion-to-electron temperature ratio from SolEdge2D-EIRENE.

\section{Modeling of a generic light impurity}

\subsection{Impurity poloidal and outer midplane distribution}

The multi-fluid nature of S2DE allows the modeling of light impurities without having to enforce the so-called trace approximation, and enables the calculation of the forces that main plasma and impurities exert on each other. This capability was recently enriched with the adoption of a new collisional 
closure [8] using the equations proposed by Zhdanov in his book [7] to express collisional closure terms such as parallel heat conductivities, collisional forces or thermal equipartition coupling sources. In fact, while the radial, cross-field transport is ruled by mean drifts and turbulence, collisional processes are of great importance for the parallel transport, especially in SOL plasmas. In the case of this particular work we assume $\mathrm{O}$ as a proxy for various light impurities $(\mathrm{C}, \mathrm{N}, \mathrm{O}, \mathrm{B}, \ldots)$ : $\mathrm{O}$ is intrinsically present because of both native oxide layers and co-deposited layers on the metallic PFCs and, together with carbon $(\mathrm{C})$, it is suspected to be one of the main sputtering species in early WEST L-mode plasma discharges [17]. As anticipated in section $3, n_{\mathrm{O}^{\mathrm{n}+}}^{\mathrm{IB}}=0.02 n_{\mathrm{D}^{+}}^{\mathrm{IB}}$ is fixed as condition at the inner boundary of the simulation domain. Then $\mathrm{O}^{\mathrm{n}+}$ ions are transported under the abovementioned diffusion coefficients, the same as for $\mathrm{D}^{+}$, while interacting with the background plasma. The resulting poloidal map of $n_{\mathrm{O}^{\mathrm{n}+}}$ is shown in figure 8.a: globally, $\mathrm{O}^{\mathrm{n}+}$ ions are found in the LFS SOL and in the lower inner divertor region, besides a fairly uniform distribution in the edge plasma. Two peculiar features are the clear effect of the second separatrix, connecting oxygen ions that reach the far SOL the upper divertor, and the depletion of the lower outer divertor region, discussed below. Equally important is to look at the poloidal map of the total $\mathrm{O}$ ion concentration, defined here as $c_{\mathrm{O}^{\mathrm{n}+}}=n_{\mathrm{O}^{\mathrm{n}+}} /\left(n_{\mathrm{D}^{+}}+n_{\mathrm{O}^{\mathrm{n}+}}\right)$ and shown, in logarithmic scale, in figure $8 . \mathrm{b}$ : it is eye catching how $c_{\mathrm{O}^{\mathrm{n}}}$ increases moving radially from the LFS separatrix $(\simeq 5 \%)$ to more remote LFS areas $(5 \%->10 \%)$ where less and less $\mathrm{D}^{+}$ ions are found. Conversely, in the edge region as well in the HFS SOL and at the two divertors $c_{\mathrm{O}^{\mathrm{n}}+}<5 \%$. By zooming in on the lower and upper divertor (figures 8.c and 8.d, respectively) it is possible to appreciate poloidally non symmetric $c_{\mathrm{O}^{\mathrm{n}}+}$ patterns. In particular, the outer divertor region is characterized by $c_{\mathrm{O}^{\mathrm{n}+}}<1 \%$, whereas the inner divertor one exhibits $c_{\mathrm{O}^{\mathrm{n}+}} \simeq 1 \%-2 \%$. Such an asymmetry, which for the upper divertor is reversed with overall higher $c_{\mathrm{O}^{\mathrm{n}}}$ values at the targets, can be explained by analyzing the balance of the parallel forces acting on $\mathrm{O}^{\mathrm{n}+}$ ions, as discussed below. In terms of radiated power, in our S2DE simulation $\mathrm{O}$ contributes with $681 \mathrm{~kW}(\simeq 83 \%$ of the total) with a pattern that is slightly less strongly localised in the SOL $\left(f_{\mathrm{rad}}^{\mathrm{SOL}} \simeq 79 \%\right)$ when compared to $\mathrm{D}$. As the reader can see in figure $5 . \mathrm{b}$, besides a non negligible contribution from the edge, the main radiating zones are the two divertors and the HFS SOL, where the pattern from the inner divertor extends way more poloidally compared to the LFS.

In order to understand what is the most represented $\mathrm{O}$ ionisation state in 

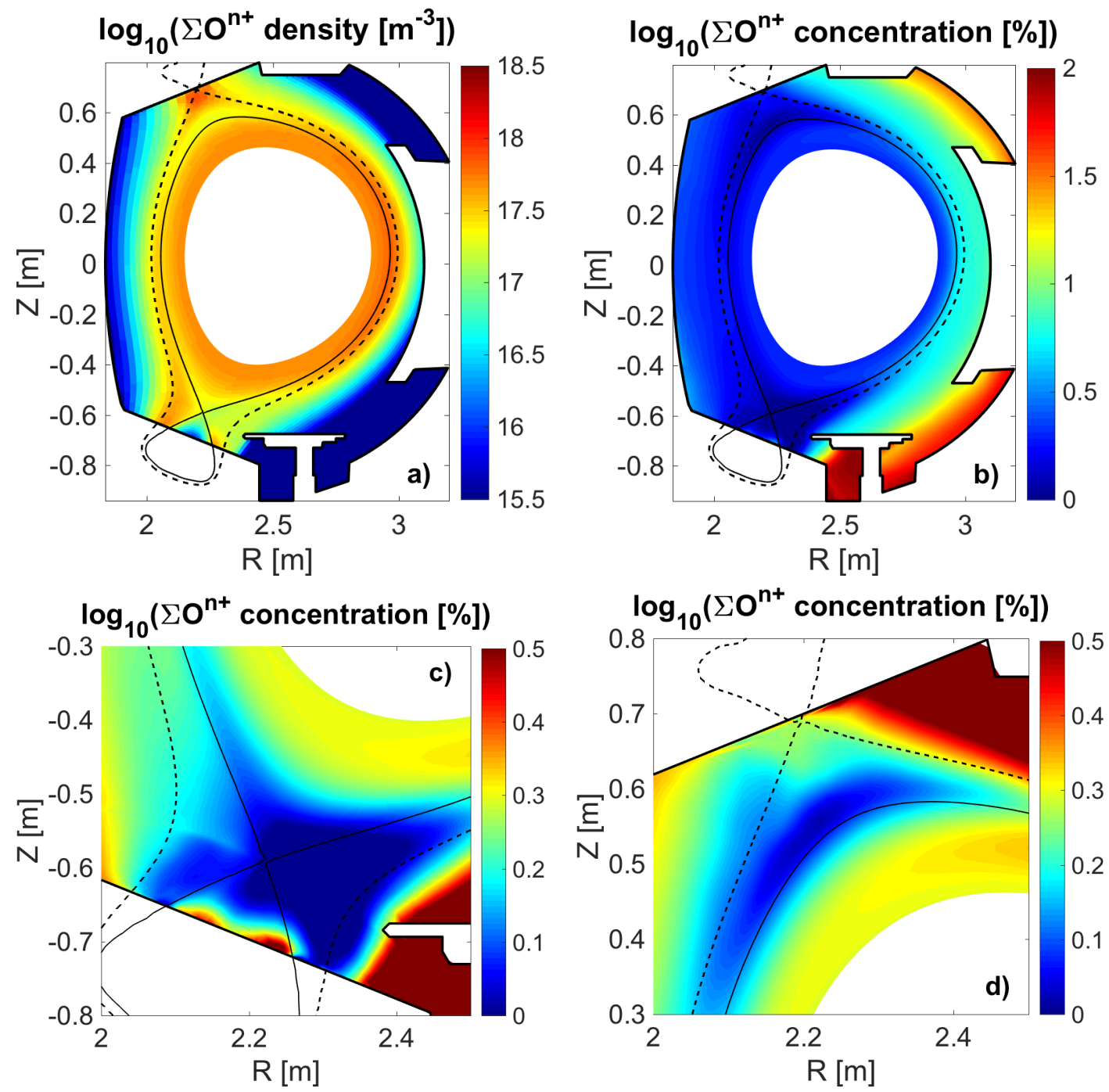

Figure 8: Poloidal maps a) total oxygen density and b) total oxygen concentration with details of the c) lower and d) upper divertor zones. Calculation from SolEdge2D-EIRENE with main (solid black line) and secondary (dashed black line) magnetic separatrix.

the high $c_{\mathrm{O}^{\mathrm{n}}}$ region at the LFS, it is useful to plot the individual density profiles along the outer midplane: in figure 9.a one can see that $\mathrm{O}^{6+}, \mathrm{O}^{7+}$, $\mathrm{O}^{8+}$ and $\mathrm{O}^{5+}$ (light blue, orange, yellow and bordeaux lines respectively) are the dominant ones along the whole profile, as one would expect in this $T_{\mathrm{e}}$ 
range from local ionisation balance, whereas the density of the lower ionisation states (not shown) is even smaller. The sum of all ionisation sates (black line) matches the prescribed value at the inner simulation boundary $\left(n_{\mathrm{O}^{\mathrm{n}+}}^{\mathrm{IB}}=0.02 n_{\mathrm{D}^{+}}^{\mathrm{IB}}\right)$ and then it peaks at $6.5 \times 10^{17} \mathrm{~m}^{-3}$ around the main separatrix like $n_{\mathrm{O}^{6+}}$, with an exponential decay in the far SOL. The corresponding individual temperature profiles along the outer midplane are shown in figure 9.b: we observe that the ionisation states populating this region are in thermal equilibrium with $\mathrm{D}^{+}$(red line, same as in figure 3.b).
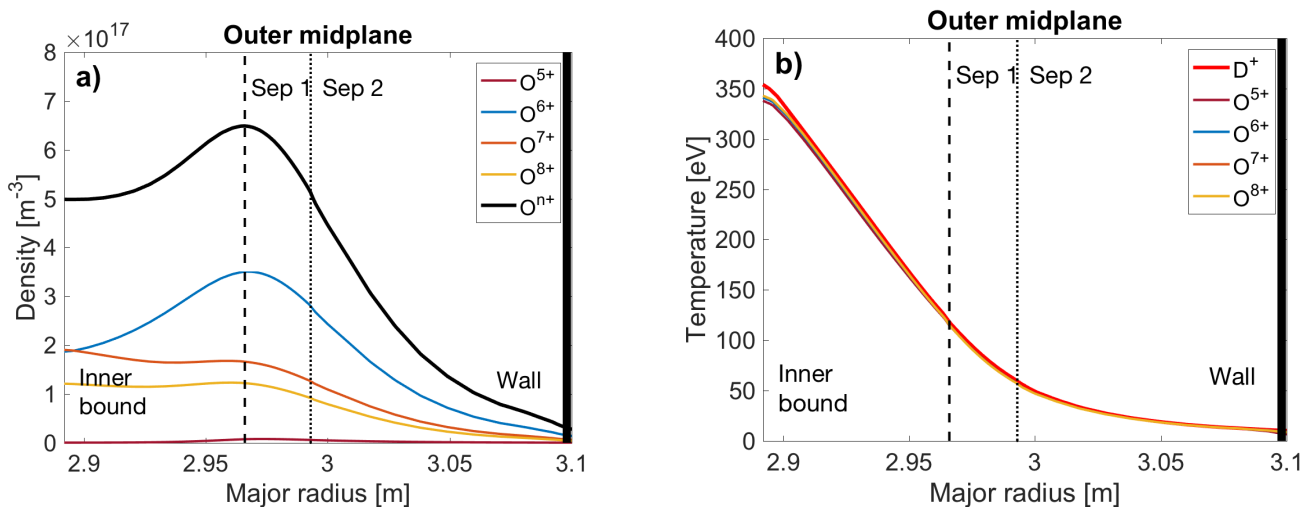

Figure 9: outer midplane radial profiles of a) density and b) temperature for oxygen high ionisation states with main (dashed black) and secondary (dotted black) separatrix and wall contour (thick black) vertical lines. Calculation from SolEdge2D-EIRENE.

\subsection{Parallel impurity distribution in the lower divertor}

Due to the strong temperature gradients between the outer midplane and the lower divertor targets, the $\mathrm{O}$ density distribution in the latter region is significantly different with respect to the former one, as expected. In figures 10.a and 10.b we show parallel profiles of density for all O ionisation states along the main magnetic separatrix in the inner and outer divertor regions, respectively. The results are qualitatively the same for the two divertor regions, with the same high ionisation states that populate the outer midplane dominating at the entrance of the divertor (right hand side of figures 10). As the divertor targets are approached (left hand side of figures 10), the density of low ionisation states becomes comparable and ultimately higher, with all 
states in the range of $10^{17} \mathrm{~m}^{-3}$, except for $\mathrm{O}^{7+}$ and $\mathrm{O}^{8+}$ (orange and yellow curves, respectively), which barely exist at these temperatures. The total $\mathrm{O}$ density (black curve), peaks in the vicinity of the divertor targets and then drops moving towards the X-point (parallel distance 5-10 m). Such drop is stronger for the outer divertor region, consistently with the $\mathrm{O}$ depletion region observed in figure 8.a and discussed below. The corresponding parallel profiles of temperature for all $\mathrm{O}$ ionisation states are presented in figures 10.c and 10.d, where $T_{\mathrm{D}^{+}}$is added as a reference (red curve). In both divertor regions, which exhibit qualitatively comparable results, the high ionisation states are in thermal equilibrium with the main ion, as already observed at the outer midplane, whereas $\mathrm{O}^{+}, \mathrm{O}^{2+}$ and $\mathrm{O}^{3+}$ (grey, purple and green curves, respectively) are $5-15 \mathrm{eV}$ colder. This discrepancy is likely due to the fact that the time needed to these ionisation states to relax through collisions towards a Maxwellian distribution with the same $T$ as the main ion is longer than their ionisation time in this temperature range.

\subsection{Impurity distribution at the wall contour}

Away from the inner simulation boundary, where the transport of $\mathrm{O}$ is governed by its interactions with the background, the density ratio $n_{\mathrm{O}^{\mathrm{n}+}} / n_{\mathrm{D}^{+}}$ can be different from $2 \%$ : at the outer strike point of our simulation, for instance, we find $n_{\mathrm{O}^{\mathrm{n}+}}^{\mathrm{OSP}} / n_{\mathrm{D}^{+}}^{\mathrm{OSP}}=0.84 \%$, whereas at the inner divertor target $n_{\mathrm{O}^{\mathrm{n}+}}^{\mathrm{ISP}} / n_{\mathrm{D}^{+}}^{\mathrm{ISP}}=1.76 \%$. These numbers indicate significant variations of the incident particle flux at the divertor plates, where light impurities govern the sputtered W flux in L-mode discharges as the one studied here. In figure 11 we present the corresponding incident particle flux along the WEST wall contour, where the key PFCs are highlighted by the grey areas: the total (ions plus neutrals) D particle flux $\Gamma_{\mathrm{D}}$ (black line) is overall two orders of magnitude higher than the total $\mathrm{O}$ particle flux $\Gamma_{\mathrm{O}^{n}}$ (blue line). Locally, though, the latter can significantly differ from $0.02 \times \Gamma_{\mathrm{D}}$ (red line). In particular, at the lower divertor targets, where both the incident particle flux and the sputtered particle flux (not shown) are peaking, one finds $\Gamma_{\mathrm{O}^{\mathrm{n}}}^{\mathrm{ISP}} / \Gamma_{\mathrm{D}}^{\mathrm{ISP}}=0.75 \%$ and $\Gamma_{\mathrm{O}^{\mathrm{n}}}^{\mathrm{OSP}} / \Gamma_{\mathrm{D}}^{\mathrm{OSP}}=0.5 \%$, respectively. Therefore, the assumption of a spatially uniform impurity concentration throughout the whole simulation domain, as done both in previous studies [24] and in the companion paper [12], represents a potentially important source of uncertainty on the calculation of sputtered fluxes using numerical models like ERO2.0 [13].

The above-mentioned strong in/out asymmetry in $\mathrm{O}$ concentration, and 

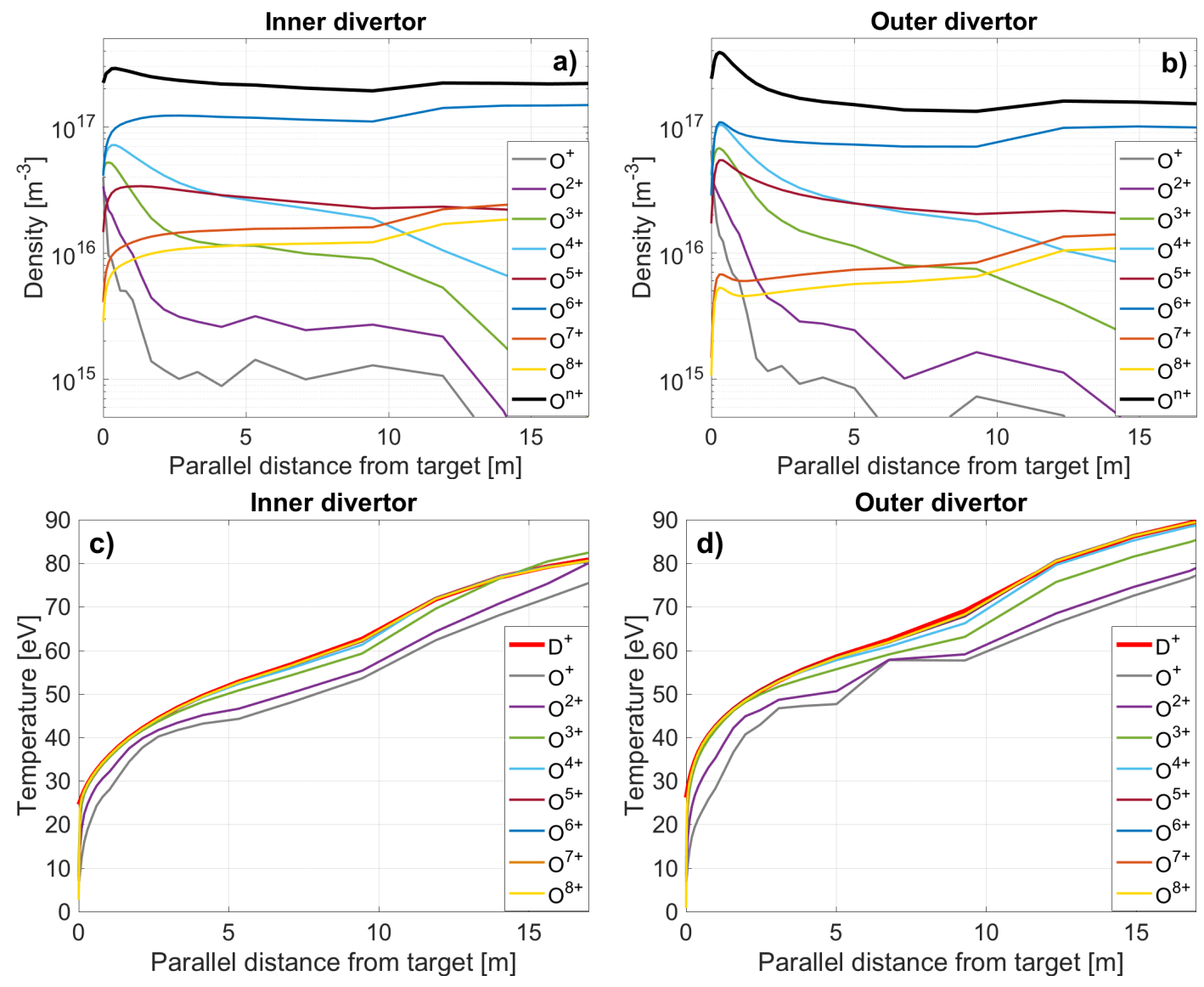

Figure 10: Parallel profiles of a),b) density and c),d) temperature for all oxygen ionisation states in inner and outer divertor, respectively. Calculation from SolEdge2D-EIRENE along the main magnetic separatrix.

hence in particle flux, observed in the lower divertor of our S2DE simulation can be explained by the balance between two main forces acting on impurities as these are among the outputs of the Zhdanov model: i) friction forces between $\mathrm{O}^{\mathrm{n}+}$ and both electrons and main ions, ii) forces due to the electron and main ion thermal gradients. Figure 12 represents these forces along a flux surface comprised between the main and the secondary separatrix, therefore in the near SOL. The zero of the horizontal axis corresponds to the inner divertor target, the other end to the outer divertor target. In the left portion of the viewgraph, if a force is positive it means it is directed towards the inner divertor target. In the right hand side, instead, a positive 


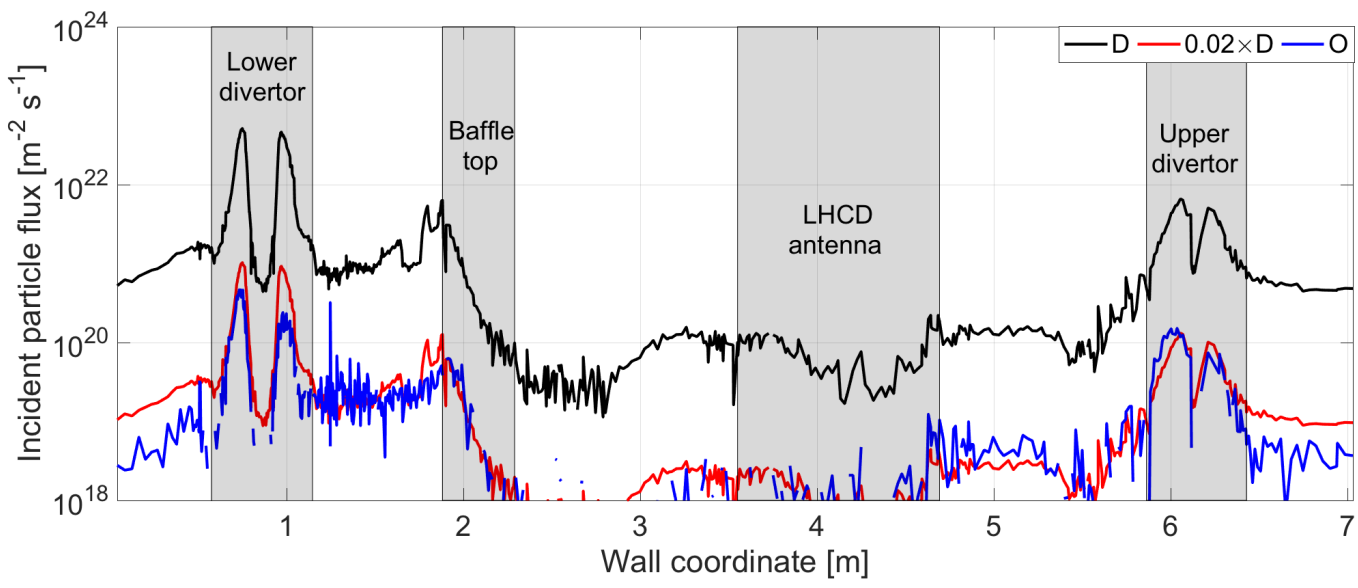

Figure 11: Wall contour profiles of the total deuterium flux (black line), $2 \%$ of the total deuterium flux (red line) and total oxygen flux (blue) with the most relevant WEST plasma-facing components (grey areas). Calculation from SolEdge2D-EIRENE.

force is a force directed away from the outer divertor target. When detailing the contribution of the different forces it appears that the friction with $\mathrm{D}^{+}$ (solid red line) flushes $\mathrm{O}$ down towards both divertor targets, whereas the deuteron thermal gradient force (blue solid line) pulls $\mathrm{O}$ away from the lower divertor. The two corresponding electron forces (dashed lines) have similar trends but are characterised by values smaller by one order of magnitude. The overall net force acting on $\mathrm{O}$ ions (black solid line) is in favour of the thermal gradient forces at both divertor targets but it is $\simeq 3$ times stronger at the outer one, resulting in the depletion of the $\mathrm{O}$ concentration in this region as observed in figure 8.a and 10.b.

\subsection{Comparison with VUV spectroscopy measurements}

The O spatial distribution obtained by S2DE can be compared with experimental data from the WEST vacuum ultraviolet spectrocopy system (VUV) [11]. The VUV spectrometer is located at the LFS equatorial plane and has a single line of sight that can be scanned around the poloidal angle in such a way that its intersection with the WEST wall contour ranges between the inner midplane and the baffle. Further technical details concerning the VUV diagnostic system and the processing of the experimental data can be found 


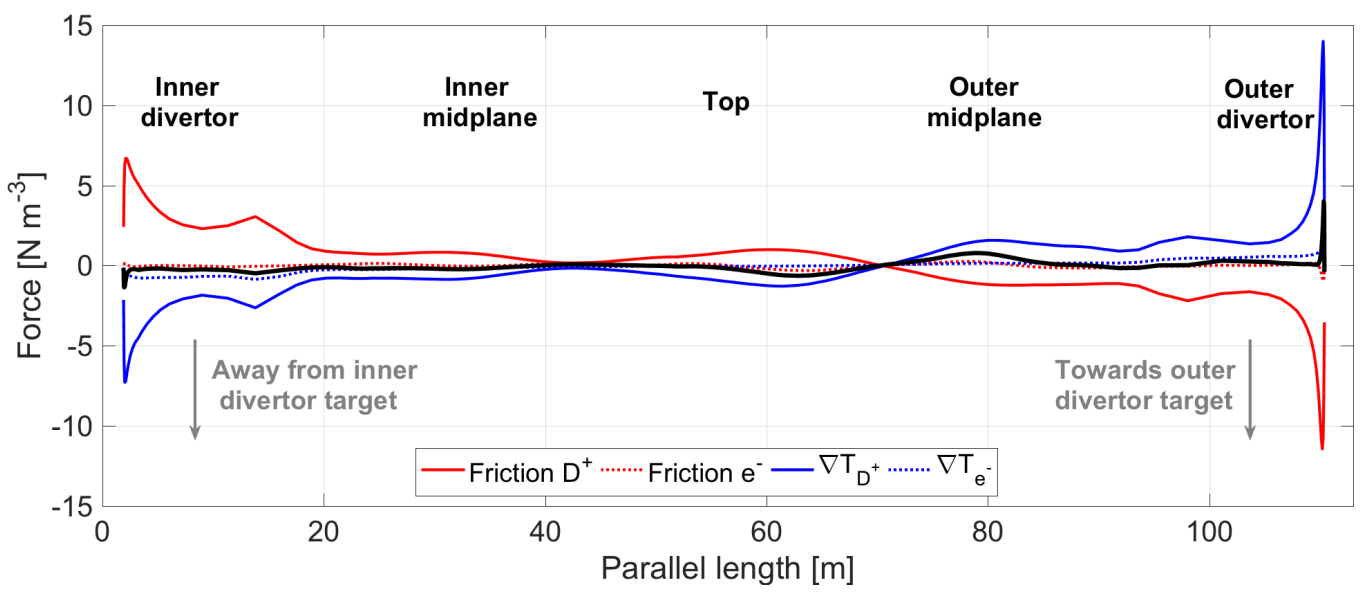

Figure 12: Parallel profile of the main forces acting on oxygen ions: friction with deuterium ions (red solid line), friction with electrons (red dashed line), deuterium ion thermal gradient (blue solid line) and electron thermal gradient (blue dashed line), net force (black thick line). Calculation from SolEdge2DEIRENE.

in the companion paper [12]. The following $\mathrm{O}^{2+}$ and $\mathrm{O}^{3+}$ lines (OIII and OIV in spectroscopic notation) are identified in the WEST VUV spectra of discharge \#54067: OIII 599.6 А, OIII 610.4 $\AA$, OIV 609.8 A, OIV 625.1 , OIV $625.9 \AA$. Since the VUV spectroscopy data are not absolutely calibrated, the experimental poloidal profiles can provide a relative information concerning the $\mathrm{O}$ light emission at the different poloidal angles and integrated along the line of sight, but cannot be used to quantify the emissivity. Therefore, both VUV data and S2DE results are normalised to their maximum value for the sake of this comparison. For a given impurity $Z$ emitting a spectral line at the wavelength $\lambda$, the emissivity $\epsilon_{\lambda}=n_{\mathrm{e}} n_{\mathrm{Z}} \mathrm{PEC}_{\lambda}$ is calculated over the whole simulation domain using poloidal maps of $n_{\mathrm{e}}, n_{\mathrm{Z}}$ provided by S2DE and the photon emission coefficient of this particular line $\mathrm{PEC}_{\lambda}$. The latter is found in the ADAS database in the form of $\mathrm{PEC}_{\lambda}\left(n_{\mathrm{e}}, T_{\mathrm{e}}\right)$ matrices and is then interpolated over the poloidal maps of $n_{\mathrm{e}}$ and $T_{\mathrm{e}}$ from S2DE using a spline method. An example of such $\epsilon_{\lambda}$ map for the OIII line at $599.6 \AA$ is shown in figure 13.a. In this particular discharge, instead of scanning the full poloidal angle range $\left(\theta=9^{\circ}-28^{\circ}\right)$, only the region of the lower divertor $\left(\theta=21^{\circ}-24.2^{\circ}\right.$, magenta lines) is scanned during the steady state plateau between 6 and $9 \mathrm{~s}$ in order to increase the spatial resolution with the aim of detecting the fine 
details of the OIII and OIV line emission patterns. By integrating the value of $\epsilon_{\lambda}\left[\mathrm{ph} \mathrm{m}^{-3} \mathrm{~s}^{-1}\right]$ along the VUV line of sight and dividing by $4 \pi$ one can calculate the corresponding brightness $B_{\lambda}\left[\mathrm{ph} \mathrm{m}^{-2} \mathrm{~s}^{-1}\right]$ profiles as a function of the poloidal angle. Such profiles are presented for OIII line at 599.6 $\AA$, for the overlapping OIII and OIV lines at $610.4 \AA$ and $609.8 \AA$, and for the OIV multiplet at $625.1 \AA$ and $625.9 \AA$ in figure 13.b, 13.c and 13.d respectively. In this set of figures the VUV experimental data are represented by the black diamonds, whereas the S2DE simulation is the solid blue line. The first striking feature is that, for all three cases, the peak of the normalised brightness $B_{\lambda} / B_{\lambda}^{\max }$ calculated by $\mathrm{S} 2 \mathrm{DE}$ occurs along the lines of sight going through the inner divertor target $\left(\theta=21^{\circ}-21.5^{\circ}\right)$. This finding agrees well with VUV measurements within the $0.3^{\circ}$ instrumental uncertainty (horizontal black lines). Another common feature between the simulations and VUV data is that $B_{\lambda} / B_{\lambda}^{\max }$ is lower by a factor of $0.4-0.6$ along the lines of sight going through the outer divertor target $\left(\theta=24^{\circ}-24.2^{\circ}\right)$. This consistency concerning the in/out asymmetry of $\mathrm{O}$ in the lower divertor region give us confidence in our capability to efficiently model the spatial distribution of light impurities with S2DE and, therefore, to thoroughly estimate on which PFCs the peaking of the incident light impurity flux and of the consequent sputtered flux occurs. Nevertheless, our description requires to be further improved as the S2DE results clearly disagree with the VUV profiles in the poloidal angle range $\theta=22^{\circ}-22.5^{\circ}$, which corresponds to lines of sight going through the private flux region $(\mathrm{PFR})$, where experimental data are characterized by a third, intermediate, peak. Here, for all three cases, the simulation exhibits a much lower $B_{\lambda} / B_{\lambda}^{\max }$. This disagreement, which is still not understood at the time of writing, could be related to the absence of $E \times B$ drifts in this particular simulation: such drifts are known to recirculate particles between the two divertor legs and might enhance the transport of $\mathrm{O}^{\mathrm{n}+}$ ions in the PFR as well as around the X-point. Furthermore, the increase of the divertor density during the VUV scanning time window discussed in section 2 could represent an additional source of disagreement between the simulations, performed as a snapshot at $t=8 \mathrm{~s}$, and the experimental data, acquired over a $3 \mathrm{~s}$ time window in which the background plasma is not exactly stationary. This hypothesis has to be tested with future simulations. On the other hand, an absolute calibration of the VUV spectroscopy data will enable a quantitative comparison with S2DE calculations, enabling not only the study of the spatial distribution of the identified light impurities but also the estimation of their fractional abundance in the WEST plasma. 

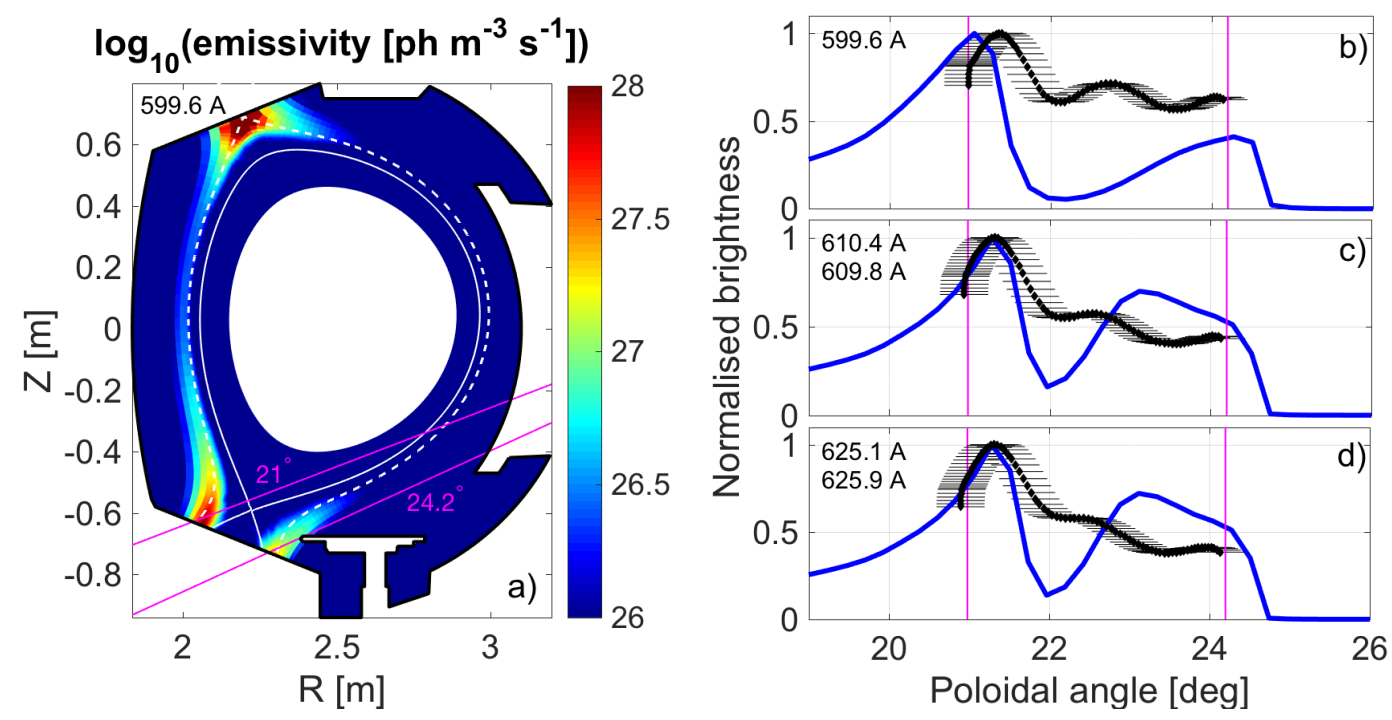

Figure 13: a) poloidal map of emissivity for the OIII line at $599.6 \AA$ from SolEdge2D-EIRENE with outermost VUV spectroscopy lines of sight (magenta lines), main (solid white line) and secondary (dashed white line) magnetic separatrix; b) angular profile of normalised brightness for OIII $599.6 \AA$, c) $610.4 \AA+$ OIV $609.8 \AA$ and d) OIV $625.1 \AA+$ OIV $625.9 \AA$ with SolEdge2D-EIRENE simulation (blue lines) and VUV spectroscopy data (black diamonds with horizontal error bars).

\section{Conclusion and future work}

In this paper we studied the transport of light impurities in the boundary plasma of a WEST discharge by interpreting a number of experimental measurements from SOL and divertor diagnostic systems with the help of the SolEdge2D-EIRENE transport code. By making experiment-based hypotheses on input power, outer midplane density and transport coefficients, we modeled a main plasma whose parameters are in good qualitative or even quantitative agreement with the measurements of a diverse set of diagnostics. This analysis allows us to check the consistency between various measurements at different locations and based on different physical phenomena, while at the same time estimating the main plasma parameters also in regions where measurements are not available. The working assumption of $\mathrm{a} \simeq 2 \%$ $\mathrm{O}$ concentration at the inner simulation boundary, as a proxy for a generic light impurity, leads to an overall underestimation of the level of radiated 
power. This discrepancy suggests that the assumed light impurity content could be too low and that, moreover, a more complex impurity mix should be considered: visible spectroscopy measurements showed that C, for instance, was one of the dominant light impurities during the WEST C3 experimental campaign, as the damage caused by isolated transient events removed part of the W coating from some carbon-based PFCs. For this reason, future modeling efforts will fully exploit the multi-fluid capabilities of SolEdge2DEIRENE to simulate a D/O/C WEST plasma with various concentrations of these impurities. Measurements of the line-integrated emission distribution of two $\mathrm{O}$ ionisation states were obtained by scanning the lower half of the plasma with the line of sight of the unique WEST VUV spectroscopy system: uncalibrated measurements of several OIII and OIV lines showed that the maximum of the $\mathrm{O}^{2+}$ and $\mathrm{O}^{3+}$ light emission occurs at the poloidal angle at which the line of sight goes through the inner divertor leg. The signal coming from the outer divertor leg is different by a factor between 0.4 and 0.6 , while a third intermediate brightness peak appears at poloidal angles corresponding to the PFR. The application of a synthetic diagnostic to the SolEdge2D-EIRENE simulation highlighted a similar O pattern, with relative brightness between inner and outer divertor in agreement with the experiment, giving us confidence regarding our capabilities of modeling the light impuritiy transport. Nevertheless, the model failed to reproduce the intermediate PFR peak. This disagreement could potentially be linked to the absence of $E \times B$ drifts in the simulation, which would modify transport in the PFR and, therefore, will be accounted for in future efforts, as already performed in [25]. Another technical limitation of this work is the fact that the assumed $O$ fraction was imposed at the inner boundary of the simulation which is found in the edge region. Coming mostly from the surface of the PFCs, O should rather be treated like a sputtered species and, therefore, generated at the wall contour. Future development efforts have to go in the direction of a more accurate mix of impurities, each of which originating from the relative $\mathrm{PFC}$ location. In this panorama, the effect of boron, an impurity which is now routinely and intentionally introduced, will have to be carefully analyzed. Another potential source of disagreement is the accuracy of the main plasma reproduced by the code: despite the overall satisfactory agreement presented in this work, further improvements will be enabled by the growing availability and reliability of measurements from the WEST diagnostic systems during more recent and future experimental campaigns. 


\section{Acknowledgements}

This work has been carried out within the framework of the EUROfusion Consortium and has received funding from the Euratom research and training programme 2014-2018 and 2019-2020 under grant agreement No 633053. The views and opinions expressed herein do not necessarily reflect those of the European Commission. Work performed under EUROfusion WP PFC.

Centre de Calcul Intensif dAix-Marseille is acknowledged for granting access to its high performance computing resources.

\section{References}

[1] V Philipps, J Roth, and A Loarte. Key issues in plasma-wall interactions for ITER: a european approach. Plasma Physics and Controlled Fusion, 45(12A):A17-A30, nov 2003.

[2] R. Dux, V. Bobkov, A. Herrmann, A. Janzer, A. Kallenbach, R. Neu, M. Mayer, H.W. Mller, R. Pugno, T. Ptterich, V. Rohde, and A.C.C. Sips. Plasma-wall interaction and plasma behaviour in the nonboronised all tungsten asdex upgrade. Journal of Nuclear Materials, 390-391:858 - 863, 2009. Proceedings of the 18th International Conference on Plasma-Surface Interactions in Controlled Fusion Device.

[3] Sebastijan Brezinsek, Andreas Kirschner, Matej Mayer, Aleksandra Wanda Baron-Wiechec, Irina Borodkina, Dmitriy Borodin, Ivor H Coffey, Jan Willem Coenen, Niek den Harder, Alina Eksaeva, Christophe Guillemaut, Kalle Heinola, Alexander Huber, Valentina Huber, Martin Imrisek, Stefan Jachmich, Ewa Pawelec, Stepan Krat, Gennady Sergienko, Guy Matthews, Andy Meigs, Sven Wiesen, and Anna Widdowson. Erosion, screening, and migration of tungsten in the jet divertor. Nuclear Fusion, 2019.

[4] J. Bucalossi, M. Missirlian, P. Moreau, F. Samaille, E. Tsitrone, D. van Houtte, T. Batal, C. Bourdelle, M. Chantant, Y. Corre, X. Courtois, L. Delpech, L. Doceul, D. Douai, H. Dougnac, F. Fasse, C. Fenzi, F. Ferlay, M. Firdaouss, L. Gargiulo, P. Garin, C. Gil, A. Grosman, D. Guilhem, J. Gunn, C. Hernandez, D. Keller, S. Larroque, F. Leroux, M. Lipa, P. Lotte, A. Martinez, O. Meyer, F. Micolon, P. Mollard, E. Nardon, 
R. Nouailletas, A. Pilia, M. Richou, S. Salasca, and J.-M. Travre. The west project: Testing iter divertor high heat flux component technology in a steady state tokamak environment. Fusion Engineering and Design, 89(7):907 - 912, 2014. Proceedings of the 11th International Symposium on Fusion Nuclear Technology-11 (ISFNT-11) Barcelona, Spain, 15-20 September, 2013.

[5] H. Bufferand, B. Bensiali, J. Bucalossi, G. Ciraolo, P. Genesio, Ph. Ghendrih, Y. Marandet, A. Paredes, F. Schwander, E. Serre, and P. Tamain. Near wall plasma simulation using penalization technique with the transport code soledge2d-eirene. Journal of Nuclear Materials, 438(Supplement):S445 - S448, 2013. Proceedings of the 20th International Conference on Plasma-Surface Interactions in Controlled Fusion Devices.

[6] H. Bufferand, G. Ciraolo, Y. Marandet, J. Bucalossi, Ph. Ghendrih, J. Gunn, N. Mellet, P. Tamain, R. Leybros, N. Fedorczak, F. Schwander, and E. Serre. Numerical modelling for divertor design of the west device with a focus on plasmawall interactions. Nuclear Fusion, 55(5):053025, 2015 .

[7] V. M. Zhdanov. Transport processes in multicomponent plasma. CRC Press, 2002.

[8] H. Bufferand, P. Tamain, S. Baschetti, J. Bucalossi, G. Ciraolo, N. Fedorczak, Ph. Ghendrih, F. Nespoli, F. Schwander, E. Serre, and Y. Marandet. Three-dimensional modelling of edge multi-component plasma taking into account realistic wall geometry. Nuclear Materials and Energy, 18:82 - 86, 2019.

[9] P. C. Stangeby. The Plasma Boundary of Magnetic Fusion Devices. 2000.

[10] D. Reiter, M. Baelmans, and P. Brner. The eirene and b2-eirene codes. Fusion Science and Technology, 47(2):172-186, 2005.

[11] J. L. Schwob, A. W. Wouters, S. Suckewer, and M. Finkenthal. Highresolution duomultichannel soft xray spectrometer for tokamak plasma diagnostics. Review of Scientific Instruments, 58(9):1601-1615, 1987.

[12] A. Sepetys et al. In preparation for Nuclear Fusion. 
[13] J. Romazanov, S. Brezinsek, D. Borodin, M. Groth, S. Wiesen, A. Kirschner, A. Huber, A. Widdowson, M. Airila, A. Eksaeva, I. Borodkina, and Ch. Linsmeier. Beryllium global erosion and deposition at jet-ilw simulated with ero2.0. Nuclear Materials and Energy, 18:331 338, 2019.

[14] C. Gil, G. Colledani, M. Domenes, D. Volpe, A. Berne, F. Faisse, C. Guillon, J. Morales, P. Moreau, and B. Santraine. Renewal of the interfero-polarimeter diagnostic for west. Fusion Engineering and Design, 140:81 - 91, 2019.

[15] Blaise Faugeras, Jacques Blum, Cedric Boulbe, Philippe Moreau, and Eric Nardon. 2d interpolation and extrapolation of discrete magnetic measurements with toroidal harmonics for equilibrium reconstruction in a tokamak. Plasma Physics and Controlled Fusion, 56(11):114010, oct 2014 .

[16] J. Urban, L.C. Appel, J.F. Artaud, B. Faugeras, J. Havlicek, M. Komm, I. Lupelli, and M. Peterka. Validation of equilibrium tools on the compass tokamak. Fusion Engineering and Design, 96-97:998 - 1001, 2015. Proceedings of the 28th Symposium On Fusion Technology (SOFT-28).

[17] G.J. van Rooij et al. In press on Physica Scripta, 2020.

[18] A.V. Chankin, D.P. Coster, R. Dux, Ch. Fuchs, G. Haas, A. Herrmann, L.D. Horton, A. Kallenbach, B. Kurzan, H.W. Mller, R. Pugno, M. Wischmeier, and E. Wolfrum and. Simulation of ASDEX upgrade ohmic plasmas for SOLPS code validation. Nuclear Fusion, 49(1):015004, dec 2008.

[19] F. Clairet, S. Heuraux, C. Bottereau, D. Molina, L. Ducobu, F. Leroux, and A. Barbuti. Fast sweeping reflectometry upgrade on tore supra. Review of Scientific Instruments, 81(10):10D903, 2010.

[20] J.P. Gunn, C. Boucher, M. Dionne, I. uran, V. Fuchs, T. Loarer, I. Nanobashvili, R. Pnek, J.-Y. Pascal, F. Saint-Laurent, J. Stckel, T. Van Rompuy, R. Zagrski, J. Admek, J. Bucalossi, R. Dejarnac, P. Devynck, P. Hertout, M. Hron, G. Lebrun, P. Moreau, F. Rimini, A. Sarkissian, and G. Van Oost. Evidence for a poloidally localized enhancement of radial transport in the scrape-off layer of the tore supra 
tokamak. Journal of Nuclear Materials, 363365:484 - 490, 2007. PlasmaSurface Interactions-17.

[21] R. Dejarnac et al. Submitted to Review of Scientific Instruments.

[22] J. Gaspar, Y. Corre, M. Firdaouss, J-L. Gardarein, D. Guilhem, M. Houry, C. Le Niliot, M. Missirlian, C. Pocheau, and F. Rigollet. Heat flux estimation in WEST divertor with embedded thermocouples. Journal of Physics: Conference Series, 745:032091, sep 2016.

[23] J. Gaspar, Y. Corre, M. Firdaouss, J.L. Gardarein, J. Gerardin, J.P. Gunn, M. Houry, G. Laffont, T. Loarer, M. Missirlian, J. Morales, P. Moreau, C. Pocheau, and E. Tsitrone. First heat flux estimation in the lower divertor of west with embedded thermal measurements. Fusion Engineering and Design, 146:757 - 760, 2019. SI:SOFT-30.

[24] A. Gallo et al. First efforts in numerical modeling of tungsten migration in west with soledge2d-eirene and ero2.0. In press, 2020.

[25] H. Bufferand, C. Baudoin, J. Bucalossi, G. Ciraolo, J. Denis, N. Fedorczak, D. Galassi, Ph. Ghendrih, R. Leybros, Y. Marandet, N. Mellet, J. Morales, N. Nace, E. Serre, P. Tamain, and M. Valentinuzzi. Implementation of drift velocities and currents in soledge2deirene. Nuclear Materials and Energy, 2017. 